\title{
MicroRNA-320a acts as a tumor suppressor in endometrial carcinoma by targeting IGF-1R
}

\author{
SHANRONG SHU ${ }^{1 *}$, XIAOPING LIU ${ }^{2 *}$, MING XU $^{3}$, XUESONG GAO $^{1}$, \\ SHU CHEN ${ }^{1}$, LAI ZHANG ${ }^{4}$ and RUIMAN LI ${ }^{1}$

\begin{abstract}
${ }^{1}$ Department of Gynecology and Obstetrics, The First Affiliated Hospital of Jinan University, ${ }^{2}$ Department of
The First Affiliated Hospital of Sun Yat-Sen University, Guangzhou, Guangdong 510080; ${ }^{4}$ Department of

Clinical Medicine, Medical College of Jinan University, Guangzhou, Guangdong 510630, P.R. China
\end{abstract} \\ Hematology, Guangzhou Institute of Pediatrics, Guangzhou Women and Children's Medical Center, Guangzhou, \\ Guangdong 510630; ${ }^{3}$ Department of Medical Ultrasound, Institute of Diagnostic and Interventional Ultrasound,
}

Received July 16, 2018; Accepted December 27, 2018

DOI: $10.3892 / \mathrm{ijmm} .2019 .4051$

\begin{abstract}
Dysregulation of microRNAs (miRs) is implicated in the carcinogenesis of various types of malignant tumor by manipulating cell growth and apoptosis. Abnormal expression of miR-320a is involved in tumorigenesis of many types of cancer. The potential association of miR-320a and the possible regulatory mechanisms in endometrial carcinoma is rarely elucidated. In the present study, it was demonstrated that miR-320a expression was decreased in endometrial carcinoma tissues and cell lines. The present results also indicated that overexpression of miR-320a suppressed cell proliferation through inducing $\mathrm{G}_{2} / \mathrm{M}$ phrase arrest and apoptosis. Insulin-like growth factor receptror-1 (IGF-1R) was verified to be the potential target of miR-320a by computational analysis and luciferase reporter assays. In addition, overexpression of miR-320a reduced endogenous IGF-1R expression in cells. Furthermore, it was demonstrated that upregulation of miR-320a inhibited phosphorylated (p)-protein kinase B and p-mechanistic target of rapamycin activation and promoted B cell lymphoma-2-associated death promoter expression. Reintroduction of IGF-1R into miR-320a-overexpressed cells antagonized the impact of miR-320a on its downstream protein, which demonstrated that the tumor suppressive role of miR-320a in endometrial carcinoma is exerted by the signal pathway mediated by IGF-1R. It was therefore concluded that
\end{abstract}

Correspondence to: Professor Ruiman Li, Department of Gynecology and Obstetrics, The First Affiliated Hospital of Jinan University, 613 Huangpu Road West, Guangzhou, Guangdong 510630, P.R. China

E-mail: butterfly1705@126.com

${ }^{*}$ Contributed equally

Key words: endometrial carcinoma, microRNA-320a, insulin like growth factor receptor-1, carcinogenesis, signal pathway
miR-320a served an anti-tumor role on endometrial carcinoma through the regulation of IGF-1R, and miR-320a may be used as the target for the gene therapy of endometrial carcinoma.

\section{Introduction}

Endometrial carcinoma is one of the most common malignant tumors in women, having the fourth highest prevalence and the eighth highest mortality rate worldwide (1). The treatment for this tumor includes surgery/chemotherapy/radiotherapy and high-dose progestational hormone (2). However, local or distant recurrences, which result from treatment-resistant cancer cells present an issue for gynecological oncologists (3). To investigate the underlying mechanisms of its carcinogenesis or progression, and to explore the potential target for an effective therapy stimulates the interests of many researchers.

MicroRNAs (miRNAs or miRs) are a group of evolutionarily conserved regulatory RNAs, which have been suggested to exert regulatory functions in carcinogenesis and used as therapeutic targets for cancer treatment (4). It has previously been reported that miRNAs exert a pivotal role in cell proliferation and metastasis in endometrial carcinoma (5). Ramón et al (6) have reported that specific miRNAs were overexpressed in endometrial carcinoma, which was positively correlated with VEGF expression. miR-200c was reported to be associated with the occurrence of endometrial carcinoma by regulating BRD7 expression (7). miR-205 was overexpressed in endometrial cancer cells and promoted tumor metastasis by downregulation of JPH4 expression (8). miR-130b (9), miR-200b (10) and miR-200 family (11) all contributed to the development of endometrial carcinoma through mediating epithelial-mesenchymal transition related protein activation. Although miRNAs act as oncogenes or anti-oncogenes in carcinogenesis and progression of endometrial carcinoma, the specific role and mechanism of miR-320a in endometrial carcinoma still remains to be elucidated.

Obesity is a high risk for endometrial carcinoma (12). Insulin is involved in the formation of obesity, and its function is exerted partly by a signal pathway mediated by insulin 
like growth factor receptor-1 (IGF-1R), as there is a cross link between insulin receptor and IGF-1R (13). To explore the potential role of miR-320a on endometrial carcinoma and clarify its underlying correlation with IGF-1R, the miR-320a expression level in human endometrial carcinoma tissues and cells was investigated, then functional analysis to detect the role of miR-320a in HEC-1A and Ishikawa cells was performed. The present results demonstrated that miR-320a was a tumor suppressor gene, which exerted anti-tumor effects through several aspects, predominantly by regulating IGF-1R expression and disturbing the phosphorylated (p)-protein kinase $\mathrm{B}$ (Akt)/p-mechanistic target of rapamycin (mTOR) signaling pathway. These results verified the impact of miR-320a on the occurrence of endometrial carcinoma, which may provide theoretical evidence for the application of target therapy.

\section{Materials and methods}

Clinical specimens and cell culture. A total of 50 endometrial carcinoma samples and 10 normal endometrium tissues were obtained from patients at the Department of Gynecology and Obstetrics of the First Affiliated Hospital of Jinan University (Guangzhou, China) from April 2015 to March 2017. Normal endometrium tissue samples were harvested from patients who experienced hysterectomy for benign uterine disease. The age of patients ranged from 42-65 years old. None of the patients had experienced chemotherapy or radiotherapy prior to surgery. All the surgically excised tissues were pathologically confirmed and stored at $-80^{\circ} \mathrm{C}$ until analysis. Ishikawa, HEC-1A, HEC-1B, HEC-251, AN3CA and RL95-2 were bought from American Type Culture Collection (ATCC, Manassas, VA, USA). Normal endometrial cells were also used. To isolate normal cells from endometrial tissues, the endometrium was rinsed 2-3 times with D-Hank's solution containing penicillin and streptomycin. Tissue samples $\left(\sim 1 \mathrm{~cm}^{3}\right)$ were administered with $2 \mathrm{ml} 0.125 \%$ trypsin. The mixture was digested in a $37^{\circ} \mathrm{C}$ incubator for $1.5 \mathrm{~h}$. The left tissue was allowed to pass through a stainless steel sieve for the second digestion. The digested cells were centrifuged at $72 \mathrm{x} g$ for $10 \mathrm{~min}$ at room temperature, and the supernatant was discarded. The cells were centrifuged twice and cultured in serum-containing medium.

All cells were cultured at $37^{\circ} \mathrm{C}$ and $5 \% \mathrm{CO}_{2}$ with Dulbecco's modified Eagle's medium (Gibco; Thermo Fisher Scientific, Inc., Waltham, MA, USA), which was supplied with $10 \%$ fetal calf serum (Hyclone; GE Healthcare Life Sciences, Logan, UT, USA) and $1 \%$ penicillin-streptomycin (Hyclone; GE Healthcare Life Sciences). The present study was performed with the approval of the Human Ethics Committee of Jinan University in accordance with the Declaration of Helsinki. All of the enrolled patients provided written informed consent.

RNA extraction and reverse transcription-quantitative polymerase chain reaction ( $R T-q P C R)$. TRIzol reagent (Invitrogen; Thermo Fisher Scientific, Inc.) was used to extract RNA from tissues and cells, then Bulge-Loop ${ }^{\mathrm{TM}}$ miRNA qRT-PCR primer kits (Guangzhou RiboBio Co., Ltd., Guangzhou, China) and All-in-One ${ }^{\mathrm{TM}}$ qPCR mix (GeneCopoeia, Inc., Rockville, MD, USA) were used to detect the expression level of miR-320a with U6 snRNA as the internal control. The primers used were as follows: miR-320a forward, 5'-ATCCAGTGCAGGGTC CGAGG-3' and reverse, 5'-CGCGGTTAAAAGCTGGGT TGAGA-3'; U6 forward, 5'-CTCGCTTCGGCAGCACA-3' and reverse, 5'-AACGCTTCACGAATTTGCGT-3'. The $2^{-\Delta \Delta C q}$ method (14) was used to determine the relative expression of miR-320a in tissues and cells.

Oligonucleotide transfection and generation of stably transfected cell lines. Cells were seeded into 6-well plates at the density of $2 \times 10^{5}$ cells/well. Lipofactamine ${ }^{\mathrm{TM}}$ RNAi MAX (Invitrogen; Thermo Fisher Scientific, Inc.) was used to transfect the cells with miR-320a mimics or controls (50 nM; Shanghai GenePharma Co., Ltd., Shanghai, China). The miR-320a mimics sequence was 5'-AAAAGCUGG GUUGAGAGGGCGA-3'. The sequence for the control was 5'-CAGUACUUUUGUGUAGUACAA-3'. Meanwhile, pcDNA-IGF-1R (100 nM; Invitrogen; Thermo Fisher Scientific, Inc.) and pcDNA-control were co-transfected using Lipofactamine 2000 reagent (Invitrogen; Thermo Fisher Scientific, Inc.). At $48 \mathrm{~h}$ following transfection, pGCSIL-GFP vector with the A-geI and EcoR I enzyme sites was inserted via the target sequences. Following recombination using pCMV and pBR322 vectors (Shanghai GeneChem Co., Ltd., Shanghai, China), lentiviral vectors (Lv) expressing targeting gene were constructed, which was simultaneously transfected into 293T cells (ATCC) coupled with packaging vectors. At $48 \mathrm{~h}$ following transfection, supernatants containing lentivirus were harvested. Ultracentrifugation $\left(4^{\circ} \mathrm{C}\right.$ for $2 \mathrm{~h} ; 210,000 \times \mathrm{g}$ ) was used to determine the titer of lentivirus.

MTT and colony formation analysis. Endometrial carcinoma cells were seeded in 96-well plates at 5,000 cells per well. MTT assay was used to determine the viability of cells at 24, 48 and $72 \mathrm{~h}$. Absorbance at $490 \mathrm{~nm}$ was determined by spectrophotometer. For colony formation assay, transfected cells were plated in culture plates and cultured for 14 days at 500 cells per plate. Colonies were fixed with methanol at room temperature for $15 \mathrm{~min}$ and stained with crystal violet at room temperature for $5 \mathrm{~min}$. The numbers of colonies were counted manually.

Western blot analysis. The total cell protein was obtained using radioimmunoprecipitation assay lysis and extraction buffer (Pierce; Thermo Fisher Scientific, Inc.) and the concentration was determined by BCA protein assay kit (Pierce; Thermo Fisher Scientific, Inc.). A total of $25 \mu \mathrm{g}$ protein was applied per lane and separated by 10-12\% SDS-PAGE, then transferred to polyvinylidene difluoride membranes (EMD Millipore, Billerica, MA, USA). Membranes were incubated overnight at $4^{\circ} \mathrm{C}$ with antibodies against IGF-1R (sc-81167; 1:1,500; Santa Cruz Biotechnology, Inc., Dallas, TX, USA), p-Akt (cat. no. 4060; 1:1,000), total (t)-Akt (cat. no. 9272; 1:1,000; Cell Signaling Technology, Inc., Danvers, MA, USA), p-mTOR (sc-101738; 1:2,000; Santa Cruz Biotechnology, Inc.), t-mTOR (sc-8319; 1:2,000; Santa Cruz Biotechnology, Inc.) and B cell lymphoma-2-associated death promoter (Bad; ab32245; 1:1,200; Abcam, Cambridge, UK). The membrane was washed and incubated with horseradish peroxidase-conjugated secondary antibody (ab205718; 

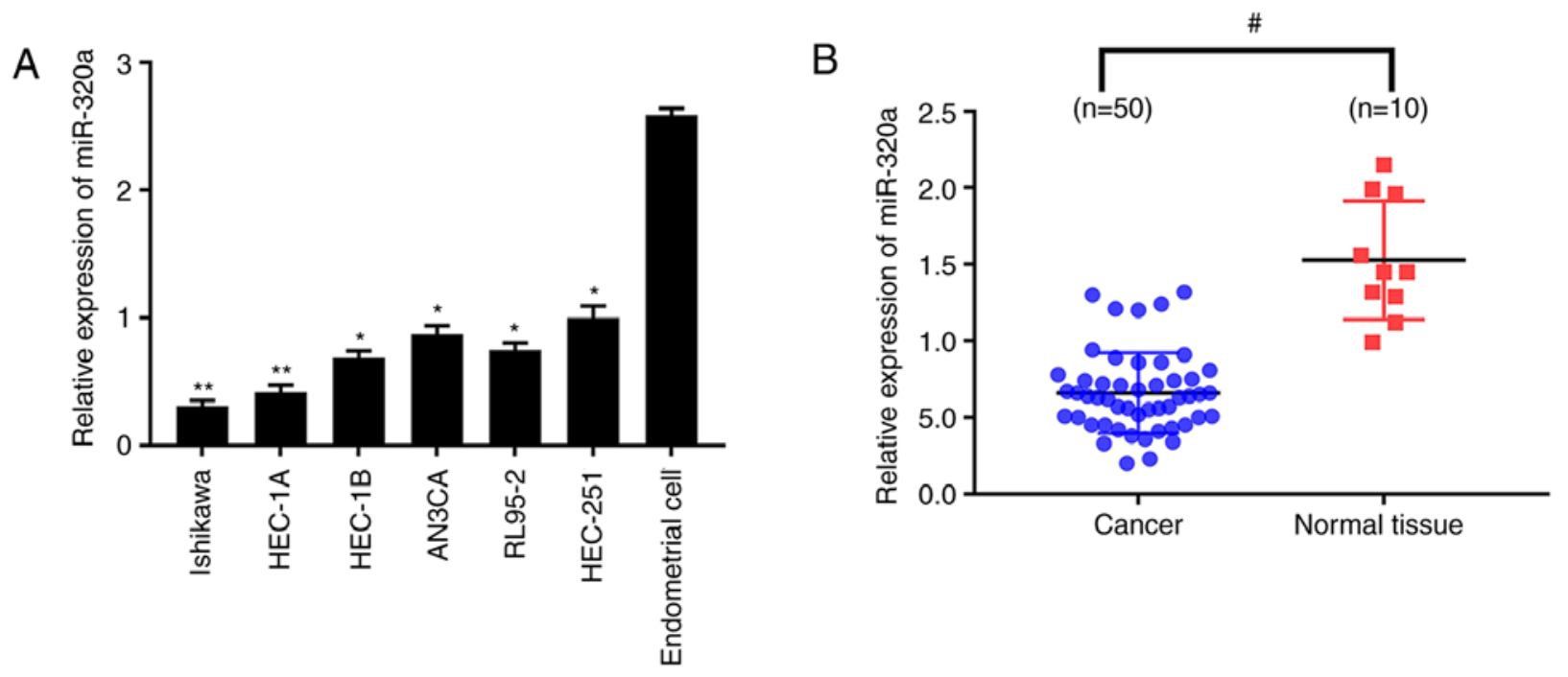

Figure 1. The expression of miR-320a was reduced in endometrial carcinoma tissue and cells. Reverse transcription-quantitative polymerase chain reaction was used to analyze miR-320a expression in (A) cell lines and (B) tissue. RNA expression was normalized with U6 snRNA. The $2^{-\Delta \Delta C 9}$ method was adopted to determine the expression of miR-320a. ${ }^{*} \mathrm{P}<0.05,{ }^{* *} \mathrm{P}<0.01$ vs. endometrial cell; ${ }^{\#} \mathrm{P}<0.05$. miR, microRNA.

1:3,000) for $1 \mathrm{~h}$ at room temperature. The protein-antibody complex was determined by an enhanced chemiluminescence system, CL-XPosure Film (Pierce; Thermo Fisher Scientific, Inc.). The relative expression of protein was quantified by Quantity One software (version 4.6.6, Bio-Rad Laboratories, Inc., Hercules, CA, USA) with GAPDH (sc-47724; 1:2,000; Santa Cruz Biotechnology, Inc.) used as the internal control. Each experiment was repeated three times to assess the consistency of the results.

Luciferase reporter assay. TargetScan database (http://www. targetscan.org/) was used to predict the possible target of miR-320a. It was demonstrated that IGF-1R may be the potential target, then the IGF-1R-untranslated region (UTR) was ligated into the pMIR-GLO luciferase vector (Promega Corporation, Madison, WI, USA) to produce recombinant pMIR-IGF-1R-UTR-Wt. Another pMIR-GLO luciferase construct containing the miR-320a mutation site in IGF-1R-UTR (CAGCUUUU to CAGCUUGU) was named IGF-1R-UTR-Mut and used as the control. Subsequently, 293T cells were simultaneously transfected with pMIR-IGF1R-UTR-Wt or pMIR-IGF1R-UTR-Mut using Lipofectamine 2000 (Thermo Fisher Scientific, Inc.). Cells were harvested following incubation at $37^{\circ} \mathrm{C}$ for $48 \mathrm{~h}$. Luciferase activity was determined and normalized with Renilla luciferase assay system (Promega Corporation).

Cell cycle distribution analysis. HEC-1A and Ishikawa cells transfected with Lv-miR-320a or Lv-control Cells were trypsinized and then the distribution of cell cycle was determined by DNA Reagent kit (Becton, Dickinson and Company, Franklin, Lakes, NJ, USA). According to the manufacturer's recommendation, the collected cells were washed and incubated with different solutions on ice in the dark for $10 \mathrm{~min}$, then flow cytometry was used to measure the relative proportions of transfected cells in different phases by FACSCalibur ${ }^{\mathrm{TM}}$ Flow Cytometry (BD Accuri ${ }^{\mathrm{TM}}$ C6; Becton, Dickinson and Company).
Detection of apoptosis by flow cytometry. Apoptosis analysis was performed according to the manufacturer's protocol (Becton, Dickinson and Company). The HEC-1A and Ishikawa cells transfected with Lv-miR-320a or Lv-control were washed and incubated at room temperature for $15 \mathrm{~min}$ with Annexin V-PE and 7-AAD in the dark. Flow cytometry was then performed to detect the apoptotic rate in the transfected cells.

Statistics analysis. SPSS 13.0 (SPSS, Inc., Chicago, IL, USA) was adopted to perform the statistical analyses. Data were expressed as the mean \pm standard deviation. Comparison between two groups was analyzed with Student's t-test, and the association between more than two groups were determined with one-way analysis of variance with Tukey's post hoc test. $\mathrm{P}<0.05$ was considered to indicate a statistically different difference.

\section{Results}

miR-320a expression is decreased in endometrial carcinoma tissues and cells. The relative expression of miR-320a among endometrial carcinoma cell lines and normal endometrial cells was quantitatively analyzed. The results demonstrated that miR-320a expression was significantly reduced in endometrial carcinoma cells compared with normal endometrial cells (Fig. 1A), which was more marked in HEC-1A and Ishikawa cell lines. miR-320a expression was then explored in 50 endometrial carcinoma and 10 normal endometrial tissues. In accordance with the results obtained from cell lines, miR-320a expression was significantly lower in endometrial carcinoma than normal endometrium (Fig. 1B). These results demonstrated that the expression of miR-320a was dramatically decreased in endometrial carcinoma.

miR-320a induces proliferation inhibition in endometrial carcinoma cells. In order to explore the role of miR-320a on cell growth, HEC-1A and Ishikawa cells were stably trans- 

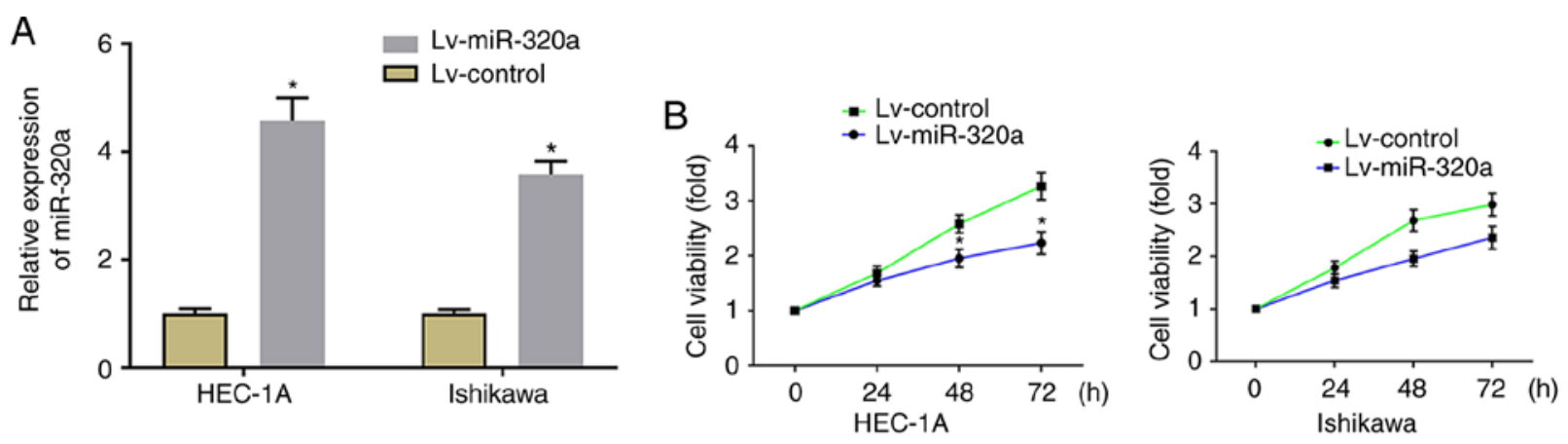

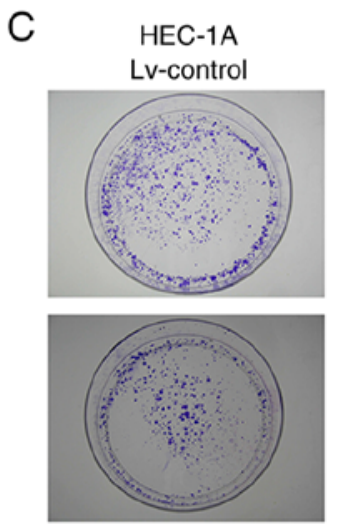

Lv-miR-320a

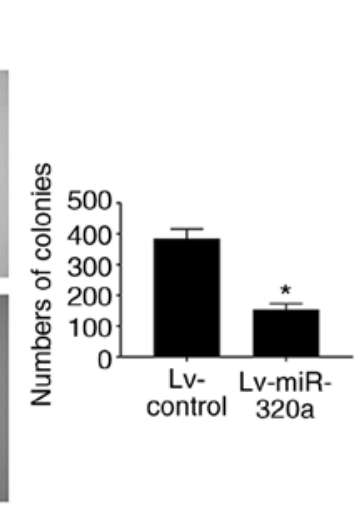

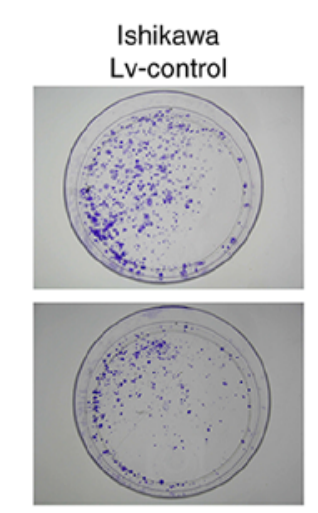

Lv-miR-320a

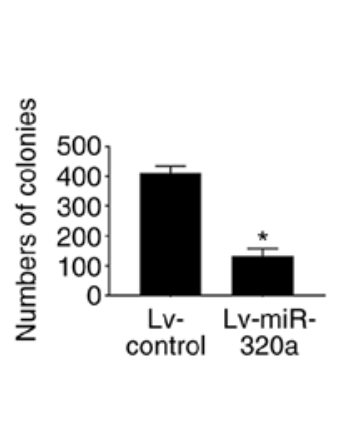

Figure 2. Upregulation of miR-320a inhibited cell growth and colony formation in HEC-1A and Ishikawa cells. (A) miR-320a expression was examined in HEC-1A and Ishikawa cells following transfection with Lv-miR-320a or Lv-control for 48 h. Overexpression of miR-320a decreased the proliferation of HEC-1A and Ishikawa cells, as demonstrated by (B) MTT analysis and (C) colony formation assay. "P<0.05 vs. Lv-control. miR, microRNA; Lv, lentivirus.

fected with Lv-miR-320a or Lv-control, then cell proliferation and colony formation ability were examined. RT-qPCR results demonstrated that miR-320a expression was significantly increased in Lv-miR-320a transfected cells, which confirmed that miR-320 was successfully transfected (Fig. 2A). As presented in Fig. 2B, MTT results demonstrated that upregulation of miR-320a significantly inhibited cell proliferation. Meanwhile, the analysis of colony formation suggested that the number of colonies was significantly decreased in Lv-miR-320a transfected cells (Fig. 2C), which verified the far-reaching role of miR-320a on cell growth.

miR-320a induces $G_{2} / M$ phase arrest and promoted cell apoptosis. It was speculated that the decreased growth in miR-320a-overexpressed endometrial carcinoma cells may result from altered cell cycle and increased cell apoptosis. To explore the present hypothesis, flow cytometry was used to detect cell cycle and apoptosis. The results demonstrated that the $\mathrm{G}_{2} / \mathrm{M}$ phases were blocked in Lv-miR-320a transfected cells, accompanied with reduction in $G_{0} / G_{1}$ phases (Fig. 3A). Meanwhile, compared with the control, it was demonstrated that miR-320a-overexpressed cells exhibited an increased apoptosis rate (Fig. 3B), which suggested that miR-320a exerts a negative mediator in endometrial carcinoma by arresting cell cycle progression and promoting cell apoptosis.

$I G F-1 R$ gene is a possible target of miR-320a in endometrial carcinoma. As miR-320a exerted an important role on endometrial carcinoma cell growth, it was inferred that the genes regulated by miR-320a may mainly function in this course.
By using the TargetScan database, it was demonstrated that the 3'-UTR of IGF-1R contains the binding site of miR-320a (Fig. 4A). Luciferase reporters were constructed to detect whether miR-320a can bind to the 3'-UTR of IGF-1R. The results demonstrated that luciferase activity in wild-type IGF-1R 3'-UTR-transfected HEC-1A and Ishikawa cells was significantly decreased by 55 and $57 \%$, respectively (Fig. 4B). However, a mutation in the miR-320a binding site to IGF-1R 3'-UTR completely eliminated this repression, which suggested that miR-320a may directly bind to this site. Consistently, overexpression of miR-320a significantly downregulated the protein expression of IGF-1R (Fig. 4C). These results suggested that the potential target of miR-320a was IGF-1R, and that miR-320a was inversely associated with IGF-1R.

Overexpression of $m i R-320$ a inhibits IGF-1R-mediated transcriptional activity. Given that IGF-1R gene was the direct target of miR-320a, it was reasonable to infer that suppression of downstream protein mediated by IGF-1R such as Akt, mTOR and Bad may be the underlying mechanism for the suppressive effect of miR-320a on cell proliferation. To verify the present assumption, western blotting was performed to examine the protein expression of $\mathrm{Akt}, \mathrm{mTOR}$ and $\mathrm{Bad}$ in cells transfected with Lv-miR-320a or Lv-control. As presented in Fig. 5, upregulation of miR-320a reduced p-Akt and p-mTOR expression with no significant effect on t-Akt or t-mTOR, but increased Bad expression. Taken together, these results suggested that miR-320a inhibited cell growth by regulating the phosphorylated protein expression mediated by IGF-1R. 

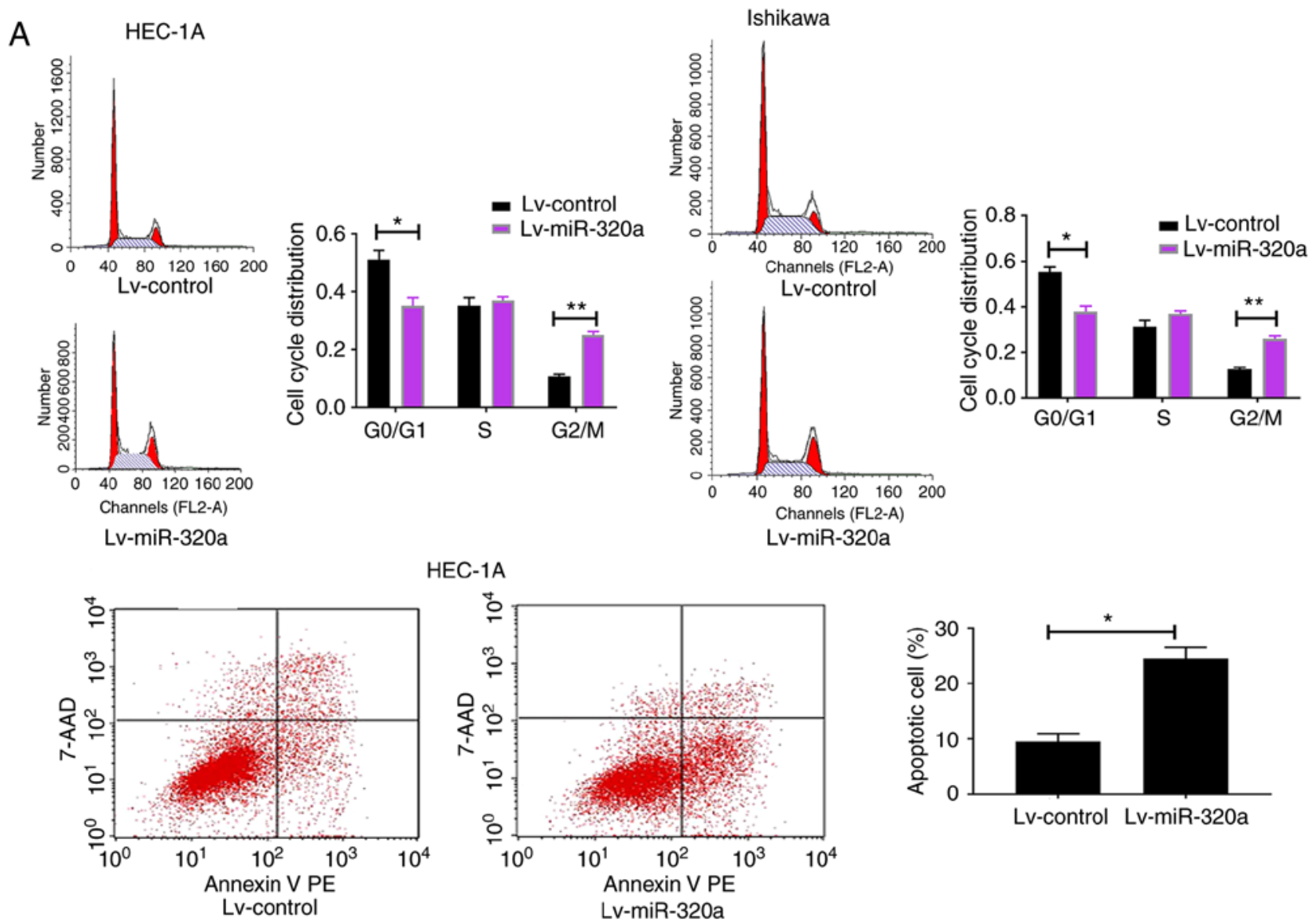

B

Ishikawa
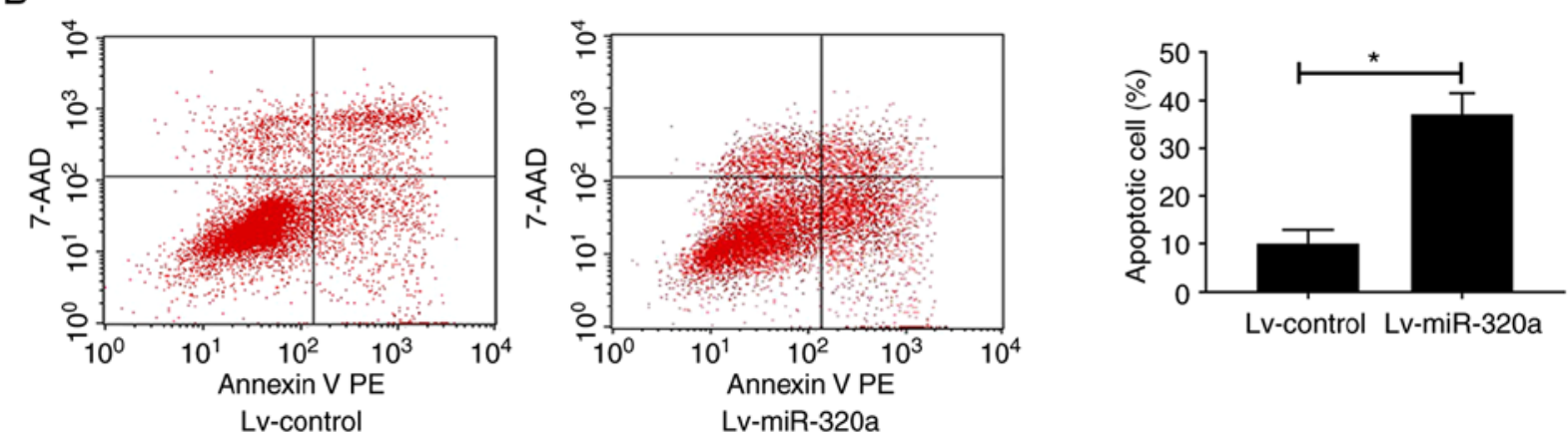

Figure 3. miR-320a blocked cell cycle progression in the $\mathrm{G}_{2} / \mathrm{M}$ phase and regulated cell apoptosis. (A) HEC-1A and Ishikawa cells were transfected with Lv-miR-320a or Lv-control for $48 \mathrm{~h}$ and cell cycle was determined by flow cytometry. Results demonstrated that $\mathrm{G}_{2} / \mathrm{M}_{\text {phase }}$ arrest and $\mathrm{G}_{0} / \mathrm{G}_{1}$ phase declined in both cell lines transfected with Lv-miR-320a. (B) Apoptosis was measured by AnnexinV-PE/7-ADD staining following Lv-miR-320a or Lv-control transfection. Results demonstrated that the rate of early and late apoptosis was increased following transfection with Lv-miR-320a. ${ }^{*} \mathrm{P}<0.05$; ${ }^{* * *} \mathrm{P}<0.01$. miR, microRNA; $\mathrm{Lv}$, lentivirus.

$I G F-1 R$ is a functional mediator for miR-320a in endometrial carcinoma. Previously, it was verified that IGF-1R was a direct target of miR-320a in endometrial carcinoma, thus it was inferred that reintroduction of IGF-1R into Lv-miR-320a transfected cells may be able to antagonize the impact of miR-320a on cell functions. To verify this assumption, IGF-1R or control were first transferred into Lv-miR-320a endometrial carcinoma cells, which was confirmed by western blotting. As presented in Fig. 6A, inreasing the expression of IGF-1R partly recovered the expression of p-Akt, p-mTOR and Bad, but had no significant impact on t-Akt and t-mTOR expression. These results demonstrated that IGF-1R was a direct mediator for miR-320a in endometrial carcinoma.

\section{Discussion}

It is proposed that miR-320a is dysregulated in many solid tumors, and its underling mechanism has been partly investigated. It has been reported that miR-320a is implicated in the metastasis of colorectal cancer to the liver (15). It has been reported that miR-320a post-transcriptionally regulated hepatocellular carcinoma migration and invasion by targeting GNAI1 (16). It was suggested that the expression pattern of miR-320a was tissue-specific and the function was cell content-dependent (17), although miR-320a was reported to modulate non-small cell lung cancer proliferation and invasion (18) and glioma cell functions (19) by directly targeting IGF-1R. Many miRNAs are 
A

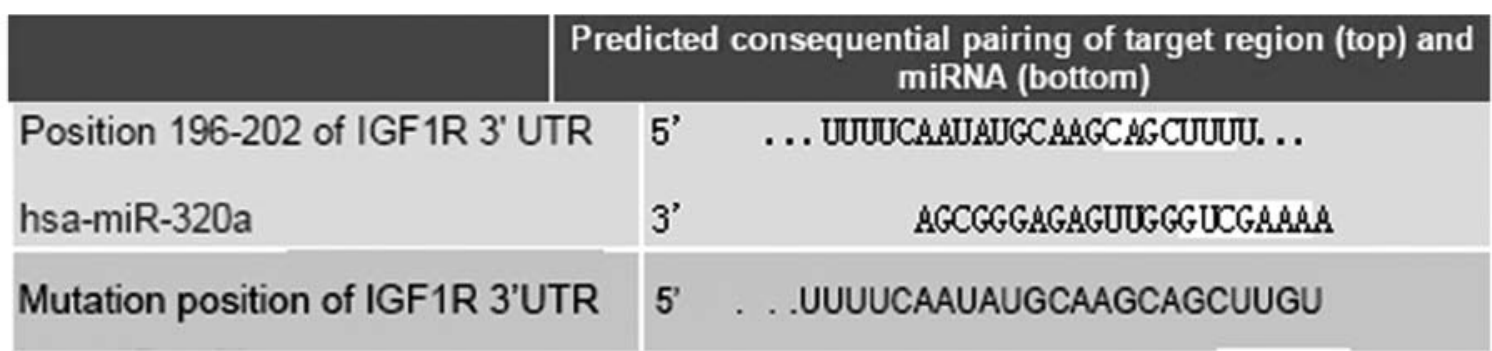

B

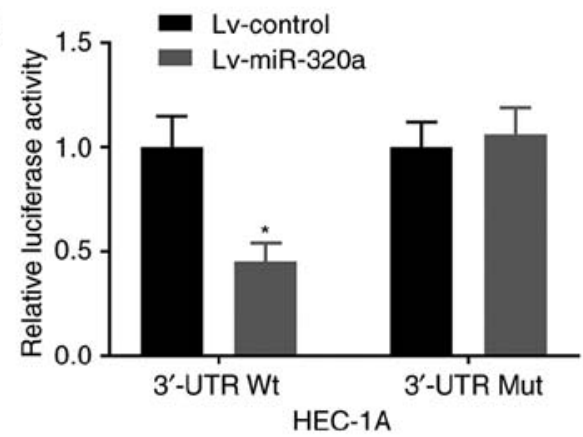

C

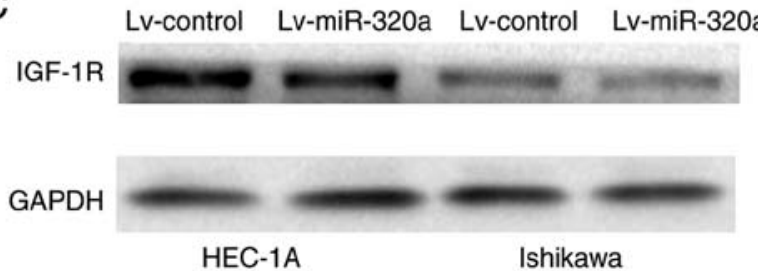

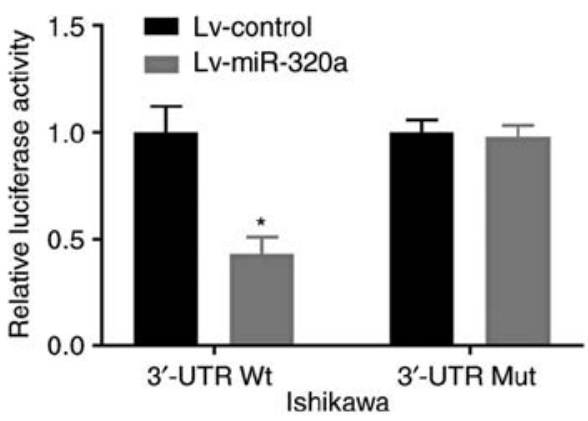

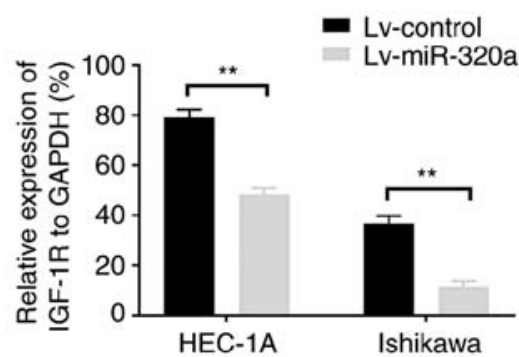

Figure 4. miR-320a directly targets IGF-1R and downregulates IGF-1R protein expression in HEC-1A and Ishikawa cells. (A) Wt and Mut potential miR-320a target sequences of IGF-1R 3'UTR. (B) Luciferase reporter assays demonstrated that miR-320a suppressed the luciferase activity of wild-type IGF-1R 3'UTR. (C) Western blotting demonstrated that miR-320a decreased IGF-1R protein expression. ${ }^{*} \mathrm{P}<0.05$ vs. Lv-control; ${ }^{* *} \mathrm{P}<0.01$. miR, microRNA; IGF-1R, insulin like growth factor receptor-1; Wt, wild type; Mut, mutant; UTR, untranslated region.

reported to have different roles in endometrial carcinoma (20), but the function of miR-320a in endometrial carcinoma and the potential mechanisms are rarely reported.

In the present research, it was demonstrated that the expression of miR-320a was dramatically decreased in endometrial carcinoma tissues and cell lines. Overexpression of miR-320a significantly inhibited cell proliferation and colony formation in HEC-1A and Ishikawa cells. Furthermore, upregulation of miR-320a lead to higher apoptosis and $\mathrm{G}_{2} / \mathrm{M}$ phrase blockage. These results suggested that miR-320a had an anti-tumor role in endometrial carcinoma. The underlying mechanism by which miR-320a exerts its impact on the progression of endometrial carcinoma was explored. It has been reported that the biological features of the specific target gene of a certain miRNA are dependent on its biological system (21). It has been reported that in leukemia cells, miR-320a increased survivin expression but in fibroblast cells, it downregulated ETS-1 expression $(22,23)$. In addition, miR-26a exerted the anti-tumor role in hepatocellular carcinoma by mediating cyclin-D2 and cyclin-E2 expression (24), but in malignant glioblastoma, miR-26a served as an oncogene by regulating PTEN function (25). So, it is important to ascertain the potential target gene of particular miRNAs in a cell-dependent pattern. In our previous study, it was demonstrated that IGF-1R is associated with the carcinogenesis and chemotherapy sensitivity of endo- metrial carcinoma $(26,27)$. It was speculated that miR-320a may function in the tumorigenesis of endometrial carcinoma through targeting IGF-1R. To detect this hypothesis, TargetScan database was used to predict the possible target gene of miR-320a, and it was demonstrated that the IGF-1R gene was the potential target of miR-320. A rescue experiment was then performed to determine whether IGF-1R is the exact regulator for miR-320a in endometrial carcinoma. The present results demonstrated that IGF-1R was the direct target of miR-320a and reintroduction of IGF-1R into miR-320a-overexpressed cells antagonized the role of miR-320a on IGF-1R downstream protein, such as p-Akt/p-mTOR and Bad. Taken together, these results suggested that IGF-1R is a functional regulator for miR-320a in endometrial carcinoma.

In conclusion, the present study demonstrated that miR-320a was an anti-tumor miRNA in endometrial carcinoma, although it is required to further investigate the role of miR-320a in the malignant transformation process from normal endometrium to proliferative or secreting phase endometrium to endometrial carcinoma. It was also demonstrated that miR-320a exerted anticancer effects through suppression of the signal pathway mediated by IGF-1R. Although miRNA-based targeted treatments are still in an initial stage, the present findings suggest that miR-320a could be an effective target for the treatment of endometrial carcinoma in future. 


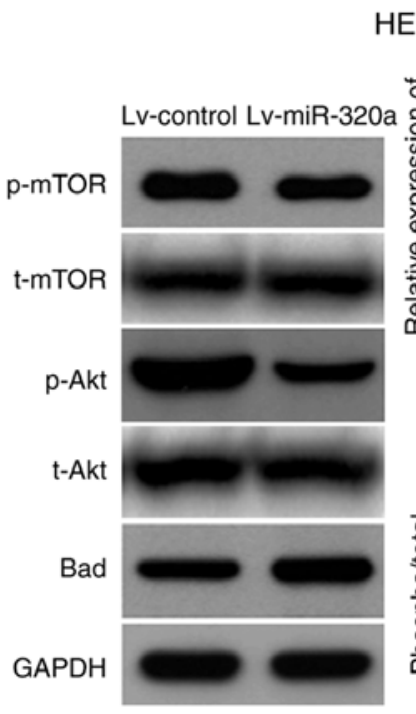

HEC-1A
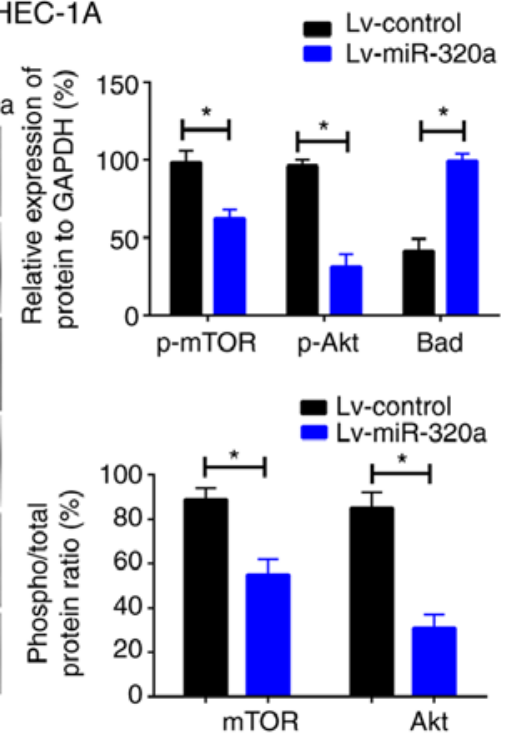

Ishikawa

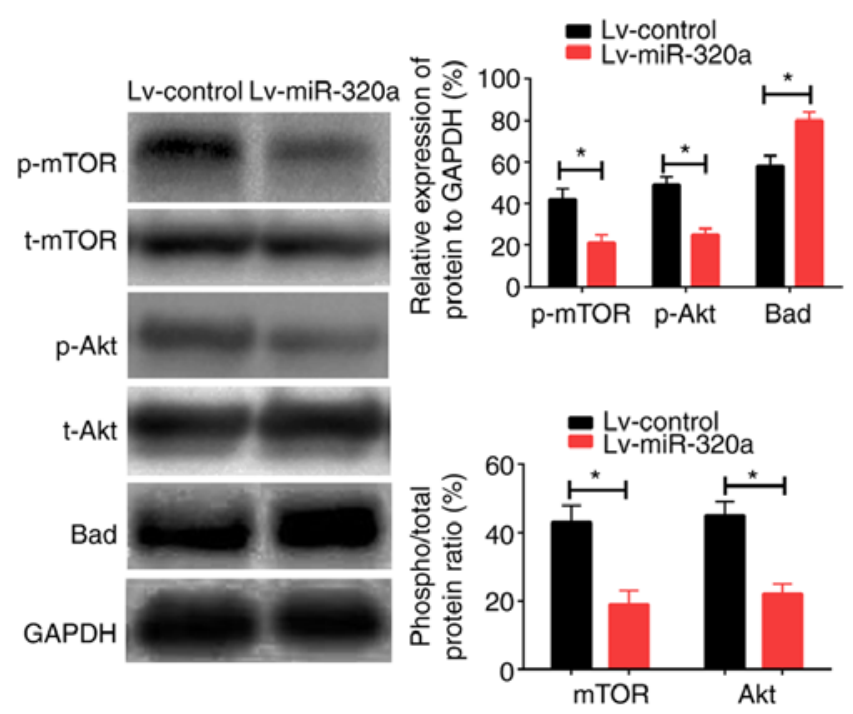

Figure 5. Western blot analysis of the expression of IGF-1R downstream protein. The results demonstrated overexpression of miR-320a significantly decreased p-mTOR and p-Akt expression and increased Bad expression, but exerted no significant effect on t-mTOR and t-Akt. Data are presented as the mean \pm standard deviation of 3 independent experiments. "P<0.05. IGF-1R, insulin like growth factor receptor-1; miR, microRNA; p, phosphorylated; mTOR, mechanistic target of rapamycin; Akt, protein kinase B; Bad, B cell lymphoma-2-associated death promoter; t, total; Lv, lentivirus.

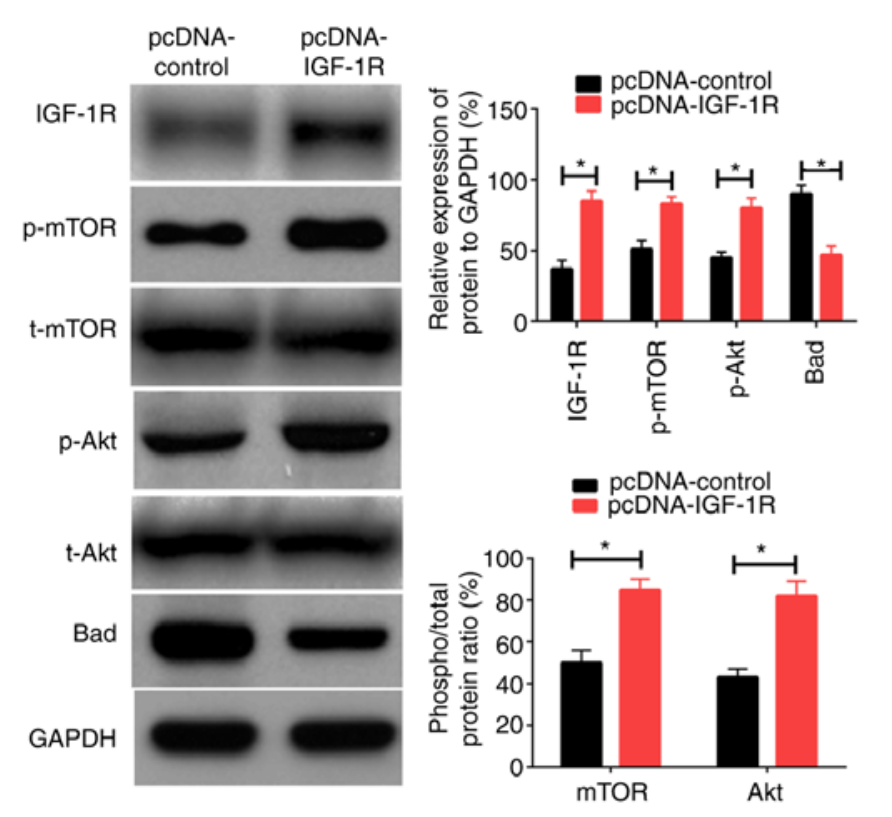

HEC-1A+Lv-miR-320a

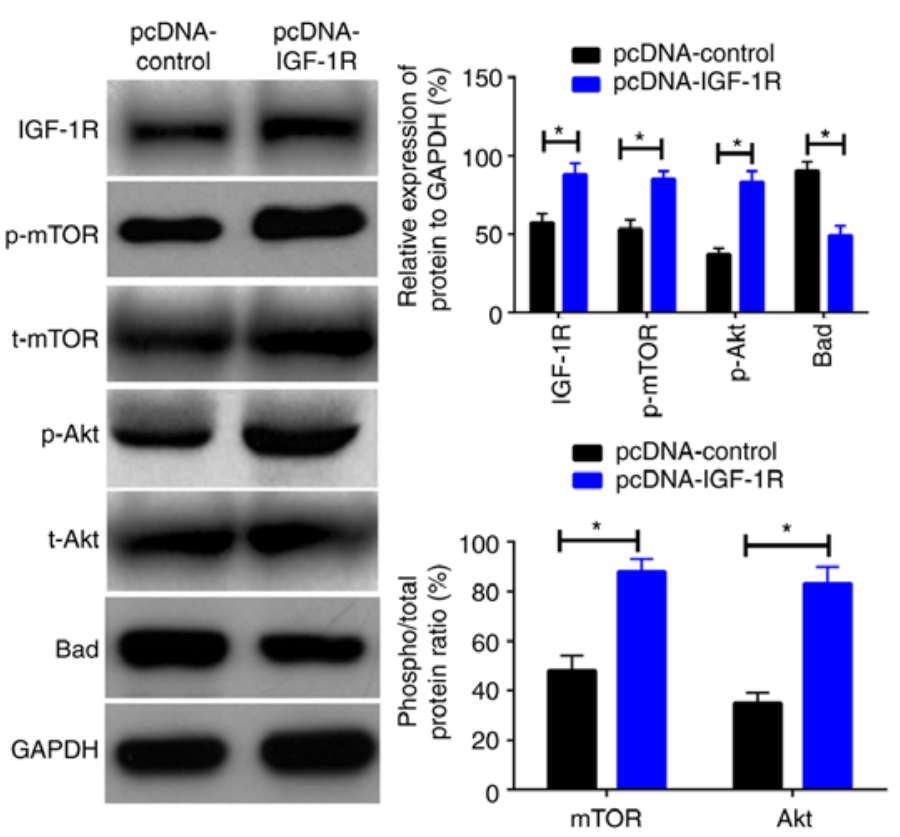

Ishikawa+Lv-miR-320a

Figure 6. The expression of related proteins mediated by IGF-1R was rescued by upregulation of IGF-1R expression. miR-320a-transfected endometrial carcinoma cells were simultaneously transfected with pcDNA-IGF-1R or control. Overexpression of IGF-1R rescued the expression of decreased p-mTOR, p-Akt and increased Bad, but had no marked effect on t-mTOR and t-Akt. Data are average value of 3 independent experiments. Data are presented as the mean \pm standard deviation of 3 independent experiments. "P<0.05. IGF-1R, insulin like growth factor receptor-1; miR, microRNA; p, phosphorylated; mTOR, mechanistic target of rapamycin; Akt, protein kinase B; Bad, B cell lymphoma-2-associated death promoter; t, total.

\section{Acknowledgements}

Not applicable

\section{Funding}

The present study was funded by National Natural Science Foundation of China (grant no. 81672496), Guangzhou Science and Technology Project (grant no. 2018040100420) and the National College Students Innovation and Entrepreneurship Training Program (grant no. CX18024).

\section{Availability of data and materials}

The authors declare that all of the data and material are freely accessible on reasonable request. 


\section{Authors' contributions}

RL and SS developed the project. MX, XG, SC and LZ performed experiments and wrote the manuscript. XL supervised the work and edit the manuscript. All authors agreed with the final version of manuscript.

\section{Ethics approval and consent to participate}

All human tissues were obtained with informed consent, and protocols were approved by the Human Ethics Committee of Jinan University (Guangzhou, China) in accordance with the Declaration of Helsinki.

\section{Patient consent for publication}

Not applicable.

\section{Competing interests}

The authors declare that they have no competing interests.

\section{Authors' information}

SS, XG, SC and RL, Department of Gynecology and Obstetrics, The First Affiliated Hospital of Jinan University, 613 Huangpu Road West, Guangzhou, Guangdong 510630, P.R. China. XL, Department of Hematology, Guangzhou Institute of Pediatrics, Guangzhou Women and Children's Medical Center, 9 Jinsui Road, Guangzhou, Guangdong 510630, P.R. China. MX, Department of Medical Ultrasound, Institute of Diagnostic and Interventional Ultrasound, The First Affiliated Hospital of Sun Yat-Sen University, 58 Zhong Shan Second Road, Guangzhou, Guangdong 510080, P.R. China. LZ, Department of Clinic Medicine, Medical College of Jinan University, 613 Huangpu Road West, Guangzhou, Guangdong 510630, P.R. China

\section{References}

1. Sanni OB, Mc Menamin ÚC, Cardwell CR, Sharp L, Murray LJ and Coleman HG: Commonly used medications and endometrial cancer survival: A population-based cohort study. Br J Cancer 117: 432-438, 2017.

2. Pautier P and Pommeret F: Systemic therapy for advanced endometrial cancer. Bull Cancer 104: 1046-1053, 2017 (In French).

3. Ballester M, Bendifallah S and Daraï E: European guidelines (ESMO-ESGO-ESTRO consensus conference) for the management of endometrial cancer. Bull Cancer 104: 1032-1038, 2017 (In French).

4. Lu J, Getz G, Miska EA, Alvarez-Saavedra E, Lamb J, Peck D, Sweet-Cordero A, Ebert BL, Mak RH, Ferrando AA, et al: MicroRNA expression profiles classify human cancers. Nature 435: 834-838, 2005.

5. Stope MB, Koensgen D, Weimer J, Paditz M, Burchardt M, Bauerschlag D and Mustea A: The future therapy of endometrial cancer: microRNA's functionality, capability, and putative clinical application. Arch Gynecol Obstet 294: 889-895, 2016.

6. Ramón LA, Braza-Boïls A, Gilabert J, Chirivella M, España F, Estellés A and Gilabert-Estellés J: microRNAs related to angiogenesis are dysregulated in endometrioid endometrial cancer Hum Reprod 27: 3036-3045, 2012.

7. Park YA, Lee JW, Choi JJ, Jeon HK, Cho Y, Choi C, Kim TJ, Lee NW, Kim BG and Bae DS: The interactions between MicroRNA-200c and BRD7 in endometrial carcinoma. Gynecol Oncol 124: 125-133, 2012.
8. Chung TK, Cheung TH, Huen NY, Wong KW, Lo KW, Yim SF Siu NS, Wong YM, Tsang PT, Pang MW, et al: Dysregulated microRNAs and their predicted targets associated with endometrioid endometrial adenocarcinoma in Hong Kong women. Int J Cancer 124: 1358-1365, 2009.

9. Li BL, Lu W, Lu C, Qu JJ, Yang TT, Yan Q and Wan XP: CpG island hypermethylation-associated silencing of microRNAs promotes human endometrial cancer. Cancer Cell Int 13: 44, 2013.

10. Dai Y, Xia W, Song T, Su X, Li J, Li S, Chen Y, Wang W, Ding H, Liu X, et al: MicroRNA-200b is overexpressed in endometrial adenocarcinomas and enhances MMP2 activity by downregulating TIMP2 in human endometrial cancer cell line HEC-1A cells. Nucleic Acid Ther 23: 29-34, 2013.

11. Snowdon J, Zhang X, Childs T, Tron VA and Feilotter H: The microRNA-200 family is upregulated in endometrial carcinoma. PLoS One 6: e22828, 2011.

12. Hendriks SH, Schrijnders D, van Hateren KJ, Groenier KH, Siesling S, Maas AHEM, Landman GWD, Bilo HJG and Kleefstra N: Association between body mass index and obesity-related cancer risk in men and women with type 2 diabetes in primary care in the Netherlands: A cohort study (ZODIAC-56). BMJ Open 8: e018859, 2018.

13. Flannery CA, Saleh FL, Choe GH, Selen DJ, Kodaman PH, Kliman HJ, Wood TL and Taylor HS: Differential expression of IR-A, IR-B and IGF-1R in endometrial physiology and distinct signature in adenocarcinoma. J Clin Endocrinol Metab 101: 2883-2891, 2016.

14. Livak KJ and Schmittgen TD: Analysis of relative gene expression data using real-time quantitative PCR and the 2(-Delta Delta C(T)) method. Methods 25: 402-408, 2001.

15. Zhang Y, He X, Liu Y, Ye Y, Zhang H, He P, Zhang Q, Dong L, Liu Y and Dong J: [Corrigendum] MicroRNA-320a inhibits tumor invasion by targeting neuropilin 1 and is associated with liver metastasis in colorectal cancer. Oncol Rep 33: 2093, 2015.

16. Yao J, Liang LH, Zhang Y, Ding J, Tian Q, Li JJ and He XH: GNAI1 suppresses tumor cell migration and invasion and is post-transcriptionally regulated by $\mathrm{Mir}-320 \mathrm{a} / \mathrm{c} / \mathrm{d}$ in hepatocellular carcinoma. Cancer Biol Med 9: 234-241, 2012.

17. van der Kolk JH, Pacholewska A and Gerber V: The role of microRNAs in equine medicine: A review. Vet Q 35: 88-96, 2015.

18. Wang J, Shi C, Wang J, Cao L, Zhong L and Wang D: MicroRNA-320a is downregulated in non-small cell lung cancer and suppresses tumor cell growth and invasion by directly targeting insulin-like growth factor 1 receptor. Oncol Lett 13: 3247-3252, 2017.

19. Guo T, Feng Y, Liu Q, Yang X, Jiang T, Chen Y and Zhang Q: MicroRNA-320a suppresses in GBM patients and modulates glioma cell functions by targeting IGF-1R. Tumour Biol 35: 11269-11275, 2014.

20. Sianou A, Galyfos G, Moragianni D, Andromidas P, Kaparos G, Baka $S$ and Kouskouni E: The role of microRNAs in the pathogenesis of endometrial cancer: A systematic review. Arch Gynecol Obstet 292: 271-282, 2015.

21. Carroll AP, Goodall GJ and Liu B: Understanding principles of miRNA target recognition and function through integrated biological and bioinformatics approaches. Wiley Interdiscip Rev RNA 5: 361-379, 2014.

22. Diakos C, Zhong S, Xiao Y, Zhou M, Vasconcelos GM, Krapf G, Yeh RF, Zheng S, Kang M, Wiencke JK, et al: TEL-AML1 regulation of survivin and apoptosis via miRNA-494 and miRNA-320a. Blood 116: 4885-4893, 2010.

23. Bronisz A, Godlewski J, Wallace JA, Merchant AS, Nowicki MO, Mathsyaraja H, Srinivasan R, Trimboli AJ, Martin CK, Li F, et al: Reprogramming of the tumour microenvironment by stromal PTEN-regulated miR-320. Nat Cell Biol 14: 159-167, 2011.

24. Chen L, Zheng J, Zhang Y, Yang L, Wang J, Ni J, Cui D, Yu C and Cai Z: Tumor-specific expression of microRNA-26a suppresses human hepatocellular carcinoma growth via cyclin-dependent and -independent pathways. Mol Ther 19: 1521-1528, 2011.

25. Huse JT, Brennan C, Hambardzumyan D, Wee B, Pena J, Rouhanifard SH, Sohn-Lee C, le Sage C, Agami R, Tuschl T and Holland EC: The PTEN-regulating microRNA miR-26a is amplified in high-grade glioma and facilitates gliomagenesis in vivo. Genes Dev 23: 1327-1337, 2009.

26. Shu S, Yang Y, Li X, Li T, Zhang Y, Xu C, Liang C and Wang X: Down-regulation of IGF-1R expression inhibits growth and enhances chemosensitivity of endometrial carcinoma in vitro. Mol Cell Biochem 353: 225-233, 2011.

27. Shu S, Li X, Yang Y,Zhang Y, Li T, Liang C and Wan J: Inhibitory effect of siRNA targeting IGF-1R on endometrial carcinoma. Int Immunopharmacol 11: 244-249, 2011. 